\title{
Absorbance detector for high performance liquid chromatography based on a deep-UV light-emitting diode at $235 \mathrm{~nm}$
}

\author{
João Flavio da Silveira Petruci ${ }^{\mathrm{a}, \mathrm{b}}$, Michael G. Liebetanz ${ }^{\mathrm{a}}$, Arnaldo Alves Cardoso ${ }^{\mathrm{b}}$, \\ Peter C. Hauser ${ }^{\mathrm{a}, *}$ \\ a Department of Chemistry, University of Basel, Spitalstrasse 51, 4056 Basel, Switzerland \\ b São Paulo State University (UNESP), Department of Analytical Chemistry, CEP 14800-970, Araraquara, SP, Brazil
}

\section{A R T I C L E I N F O}

\section{Article history:}

Received 23 May 2017

Received in revised form 6 July 2017

Accepted 9 July 2017

Available online 11 July 2017

\section{Keywords:}

Light-emitting diode

HPLC

Deep-UV

Absorbance detection

\begin{abstract}
A B S T R A C T
In this communication, we describe a flow-through optical absorption detector for HPLC using for the first time a deep-UV light-emitting diode with an emission band at $235 \mathrm{~nm}$ as light source. The detector is also comprised of a UV-sensitive photodiode positioned to enable measurement of radiation through a flow-through cuvette with round aperture of $1 \mathrm{~mm}$ diameter and optical path length of $10 \mathrm{~mm}$, and a second one positioned as reference photodiode; a beam splitter and a power supply. The absorbance was measured and related to the analyte concentration by emulating the Lambert-Beer law with a log-ratio amplifier circuitry. This detector showed noise levels of $0.30 \mathrm{mAU}$, which is comparable with our previous LED-based detectors employing LEDs at 280 and $255 \mathrm{~nm}$. The detector was coupled to a HPLC system and successfully evaluated for the determination of the anti-diabetic drugs pioglitazone and glimepiride in an isocratic separation and the benzodiazepines flurazepam, oxazepam and clobazam in a gradient elution. Good linearities $(r>0.99)$, a precision better than $0.85 \%$ and limits of detection at sub-ppm levels were achieved.

(c) 2017 Elsevier B.V. All rights reserved.
\end{abstract}

\section{Introduction}

Optical detection with light-emitting diodes (LEDs) is highly useful in analytical chemistry. From the introduction of the first LED emitting red light in the late 1960s to the current period - where the UV, visible and IR spectral ranges can be covered with emission bands of typically $30 \mathrm{~nm}$ width - the development of many optical detection devices has been driven by the availability of new LEDs as light sources [1-4]. Besides their small emission bandwidths, which eliminates the need for a monochromator, there are many advantages to LEDs, for instance, affordable price, compactness, robustness, flexible configuration possibilities, long lifetime and low power consumption [5]. Also, the high output stability provided by LEDs implies low noise levels and, therefore, low limits of detections are generally achieved [6]. All the aforementioned positive characteristics of LEDs have led to the development of diverse applications in the analytical chemistry field, such as in flow injection analysis [1,7], gas-sensors platforms [8], membrane-based sensors

\footnotetext{
* Corresponding author.

E-mail address: peter.hauser@unibas.ch (P.C. Hauser).
}

[9], detection in capillary electrophoresis $[6,10,11]$ and point of care devices [12,13].

Although HPLC is perhaps the most powerful and well-defined separation technique, only few studies that employ LED-based detectors coupled to the separation column have been reported up to date. Green LEDs were employed for the detection of heavy metals after ion-chromatography separation followed by reaction with PAR (4-(2-pyridylazo)resorcinol) [14,15]. Alkaline earth-metals were indirectly detected as their complexes with o-cresolphthalein after low pressure ion-chromatography using LEDs emitting at 574 and $621 \mathrm{~nm}$ [16]. Methylene blue was added to the mobile phase to create a background signal monitored by a LED-based detector emitting at $565 \mathrm{~nm}$ and used for the indirect determination of alcohols by reverse-phase liquid chromatography [17].

For HPLC, however, optical detection in the visible range is of limited use, because the majority of the potential analytes absorb only in deep-UV range $(<300 \mathrm{~nm})$. This fact helps to understand why LED-based detectors have not been widely employed for chromatographic techniques. Despite the strong development over the decades, LEDs emitting in the deep-UV range have only become available relatively recently [18]. Following their introduction, our research group reported the design of HPLC detectors based on LEDs emitting at 280 and $255 \mathrm{~nm}[19,20]$, and other groups have 
more recently also been active in this area [21-23]. The first report showed the potential of deep-UV LEDs to this application with the response in terms of transmittance rather than absorbance, hence, a signal which was not linear with concentration. A follow-up with a second device was designed with a log-ratio amplifier circuitry enabling direct absorbance responses. In both cases, the detectors were evaluated with chromatographic separations of analytes that have their maximum absorption centered at 280 and $255 \mathrm{~nm}$. Recently, Li et al. [24] have evaluated the performance of a $235 \mathrm{~nm}$ UV-LED based on-capillary photometric detector for the determination of nitrite, nitrate and iodide by capillary ion-exchange chromatography. This report was the first on the use of a deep-UV LED with shorter wavelengths than $250 \mathrm{~nm}$ for photometric detection. In this communication, we describe the use of the $235 \mathrm{~nm}$ deep-UV LED for detection of organic compounds in standard HPLC using a reversed phase column in isocratic as well as gradient elution mode.

\section{Experimental}

\subsection{Instrumentation}

The UV-LED with a nominal emission band at $235 \mathrm{~nm}$ was obtained from Crystal IS (Green Island, NY, USA) and the UVsensitive photodiodes (SG01L-C) were acquired from Sglux Sol-Gel Technologies (Berlin, Germany). The beam splitter (G344312000) was obtained from Qioptiq Photonics (Munich, Germany). The log-ratio amplifier (LOG101) and the dual operational amplifier (OPA2227) were products of Texas Instruments (Austin, TX, USA). The flow-through cuvette (76.1/SS) with dimensions of $12.5 \times 12.5 \times 45 \mathrm{~mm}$, an optical path length of $10 \mathrm{~mm}$, round aperture of $1 \mathrm{~mm}$ and nominal internal volume of $8 \mu \mathrm{L}$ was purchased from Starna Scientific (Pfungstadt, Germany). The experiments were conducted using a HPLC system (Agilent 1100) equipped with a photodiode-array detector (DAD) (Agilent, Waldbronn, Germany) using the separation column LiChrospher 60 RP-select B $(125 \times 4 \mathrm{~mm} ; 5 \mu \mathrm{m})$ from Merck (Darmstadt, Germany). Data from the LED-detector were acquired using an e-corder ED401 dataacquisition system (EDAQ, Denistone East, NSW, Australia) and the Chart software package (EDAQ) running on a personal computer. Absorption spectra were obtained with a conventional UV/Visspectrophotometer from Varian (Santa Clara, CA, USA).

\subsection{Reagents}

All chemicals employed were either analytical or HPLC grade. Acetonitrile and methanol were obtained from Avantor (Center Valley, PA, USA). Deionized water was purified using a NANOPure water purification system (Barnstead, IA, USA). Potassium phosphate monobasic $\left(\mathrm{KH}_{2} \mathrm{PO}_{4}\right)$ was obtained from Sigma-Aldrich (Buchs, Switzerland) and phosphoric acid $\left(\mathrm{H}_{3} \mathrm{PO}_{4}\right)$ was purchased from VWR (Dietikon, Switzerland). Flurazepam, oxazepam and clobazam were obtained from Sigma-Aldrich (Buchs, Switzerland). Pioglitazone hydrochloride and glimepiride were acquired from Thermofisher (Karlsruhe, Germany) and Flurochem (Hadfield, UK), respectively. Stock solutions of flurazepam, oxazepam and clobazam were prepared in acetonitrile with a concentration of $100 \mathrm{mg} \mathrm{L}^{-1}$. Pioglitazone and glimepiride stock solutions were prepared in methanol with concentrations of $500 \mathrm{mg} \mathrm{L}^{-1}$. Fresh working standard solutions were prepared daily by appropriate dilution of the stock solutions with acetonitrile.

\section{Results and discussion}

\subsection{Detector design and noise evaluation}

The arrangement used in this study was similar to the one described in our previous report [20] on the deep-UV LED based detector emitting at 280 and $255 \mathrm{~nm}$. An overall sketch and a circuitry similar to the one employed here were shown previously. The LED was driven by a constant current source to minimize fluctuations of emission intensity. The deep-UV LEDs require a relatively high current to provide best intensity - which is still low when compared to visible LEDs. The part of the electrical power which is not converted into light is turned into heat, therefore thermal management is essential to avoid overheating of the device. We employed a heat sink made from aluminium with dimensions of $18 \times 45 \times 1 \mathrm{~mm}$ to reduce the thermal resistance. Next, the light from the LED was divided by using a beam splitter positioned at $45^{\circ}$ with a splitting ratio of $80: 20 \%$, this means that one part $(80 \%)$ was passed perpendicularly through the round aperture $(1 \mathrm{~mm})$ of the flow-through cuvette, and then to the signal photodiode, while the other part (20\%) of the beam was guided to a reference photodiode. While the implementation of the referenced approach is a slight complication, this helps to compensate the temperature coefficient in intensity of about $2 \% /{ }^{\circ} \mathrm{C}$ found for the LEDs [23]. The photocurrents obtained at the photodiodes ( $i_{0}$ and $\left.i\right)$ were handled with the aid of a log-ratio amplifier circuitry, which produces an output voltage $\left(V_{O}\right)$ following the equation $V_{O}=\log \left(i_{0} / i\right)$, where $1 \mathrm{~V}$ equals to 1 absorbance unit (AU). The output was processed with an offset circuitry based on an operational amplifier to compensate for an imbalance between the intensities of the two signals, i.e. to zero the absorbance reading, and an active dual-pole low pass filter with a cut-off frequency of $10 \mathrm{~Hz}$, also based on an operational amplifier, to reduce high-frequency noise. No further noise filtering was employed. The UV- LED was mounted on a micromanipulator stage in order to allow its alignment in front of the window of the flow-through cuvette such that maximum intensity is obtained on the signal photodiode. All components, including the flow-though cuvette, mechanical parts and the electronic circuitry, were placed in a grounded metal box with dimensions of $140 \times 100 \times 73 \mathrm{~mm}$ $(\mathrm{L} \times \mathrm{W} \times \mathrm{H})$.

The UV-LED was driven with a constant current of $66 \mathrm{~mA}$. The photocurrents on the signal and reference photodiodes were measured as $18 \mathrm{nA}$ and $46 \mathrm{nA}$ respectively. Firstly, the electronic noise was evaluated by recording the baseline signal for $60 \mathrm{~s}$ and determining the maximum deviations for this period. A background noise of $0.30 \mathrm{mAU}$ and for the LED emitting at $235 \mathrm{~nm}$ was found, which is higher than the value of $30 \mu \mathrm{AU}$ reported by Li et al. [24]. However, please note that, the latter value was obtained for a significantly more agressive low pass filter setting $(0.3 \mathrm{~Hz}$ cut-off instead of $10 \mathrm{~Hz}$ ). As illustrated by Bui and Hauser for UV-LEDs, the determination of the noise is very much affected by the amount of electronic filtering applied as well as the method used for its quantification [6]. The baseline drift over a period of $12 \mathrm{~min}$ was determined as 1.1 mAU, which is comparable to our previous finding with deepUV LEDs [20].

\subsection{Evaluation of HPLC performance}

The UV-LED based detector was evaluated in HPLC separations using standard solutions of two different classes of organic compounds with absorption bands in the low UV-range. We selected two anti-diabetic drugs - pioglitazone and glimepiride - for separation under isocratic conditions and three benzodiazepine compounds, namely flurazepam, oxazepam and clobazan, to separate in gradient elution mode. Separations parameters were adapted from previous published reports $[25,26]$. The absorption 


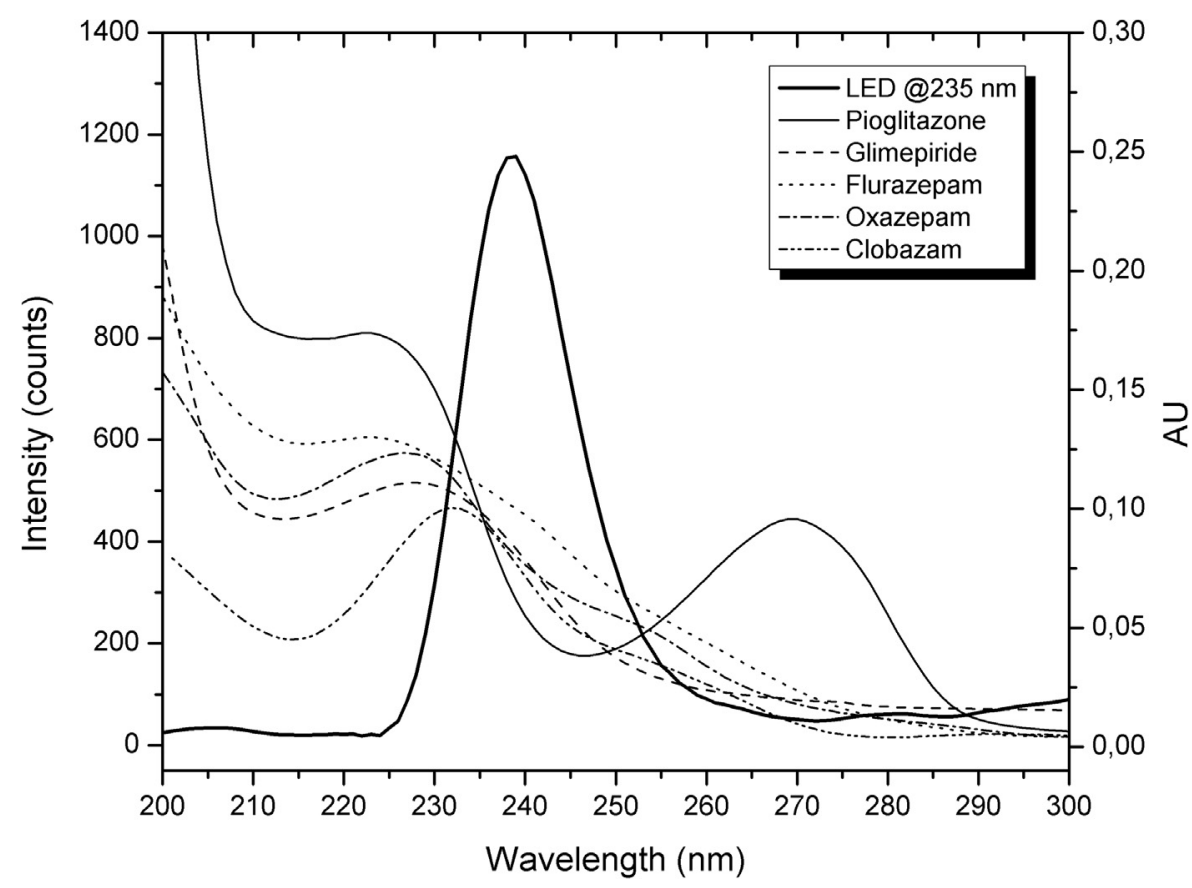

Fig. 1. Absorbance spectra of the compounds (right hand scale) and emission spectrum of the UV-LED (left hand scale).

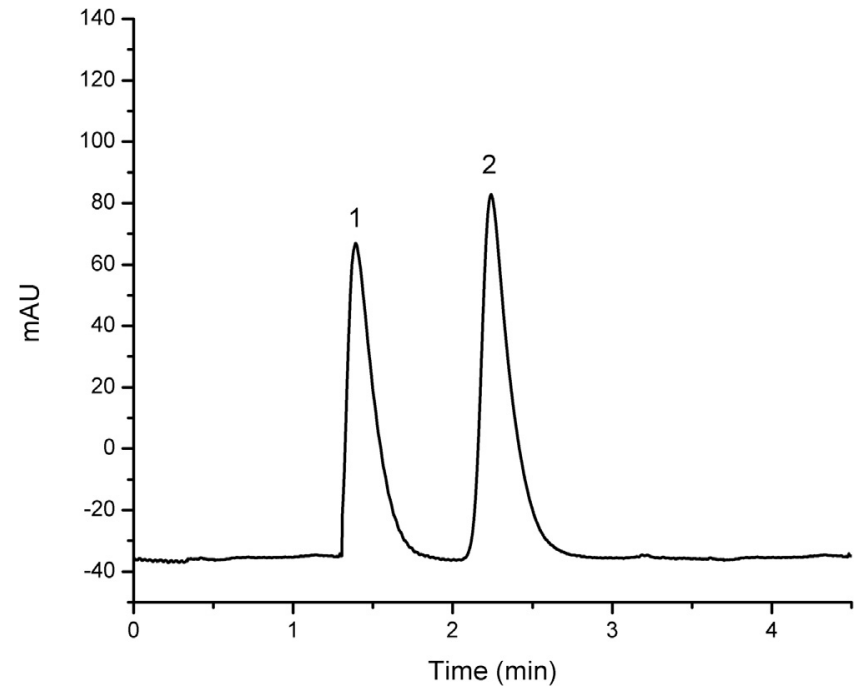

Fig. 2. Separation of two anti-diabetic drugs with detection at $235 \mathrm{~nm}$. Standard solution of $50 \mathrm{mg} \mathrm{L}^{-1}$ for each analyte: (1) Pioglitazone; (2) Glimepiride; Column:

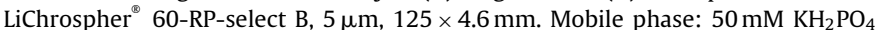
( $\mathrm{pH} 3.1$ with $\mathrm{H}_{3} \mathrm{PO}_{4}$ )/ACN 40:60 (v/v). Flow rate: $1.2 \mathrm{~mL} \mathrm{~min}^{-1}$. Injected volume: $8 \mu \mathrm{L}$.

spectra of all compounds are shown in Fig. 1 as well as the emission spectrum of the LED. Note that the peak of the emission band was found to be at $238 \mathrm{~nm}$, i.e. at a slightly longer wavelength than the nominal $235 \mathrm{~nm}$. With the exception of pioglitazone, which also shows an absorption band at $270 \mathrm{~nm}$, all species display increasing absorptivities below $255 \mathrm{~nm}$, the lowest wavelength previously accessible with UV-LEDs, which therefore endorses the application of the new LED.

The anti-diabetic drugs were separated using isocratic elution

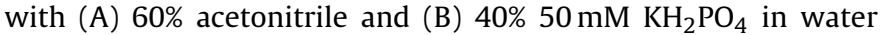
( $\mathrm{pH}=3.1$ adjusted with $\mathrm{H}_{3} \mathrm{PO}_{4}$ ). The total running time was 5 min with a flow rate of $1.2 \mathrm{~mL} \mathrm{~min}^{-1}$. The chromatogram of a standard solution of pioglitazone and glimepiride is shown in Fig. 2. Baseline deviation for isocratic condition was found to be better than
$0.35 \mathrm{mAU}$, indicating suitable stability of the emitted light provided by the deep-UV LED detector at this short wavelength. Quantitative data were calculated in order to evaluate the detector performance, where calibration curves were established based on the relation between peak area and the analyte concentration. As can be seen by the correlation coefficients in Table 1, excellent linearity was achieved for both compounds $(r>0.999)$ over the concentration range of 5-100 $\mathrm{mg} \mathrm{L}^{-1}$ for each analyte. Note, that Lambert-Beer's law is valid strictly only for monochromatic light and thus deviations from non-linearity could be expected. However, as discussed for the UV-LEDs by Bomastyk et al., for small changes of absorptivity for the compounds across the wavelengths of the emission band of the LED linearity may be approximated, at least for a narrow absorbance range [20]. A reproducibility of better than $0.8 \%$ (RSD, $n=5$ ) was achieved. The limits of detection - represented as the concentrations corresponding to peaks whose heights are three times the baseline noise - are also summarized in Table 1 .

The separation of the benzodiazepines was performed using the following chromatographic conditions: mobile phase: (A) $50 \mathrm{mM}$ $\mathrm{KH}_{2} \mathrm{PO}_{4}$ in water $\left(\mathrm{pH}=3.1\right.$ adjusted with $\left.\mathrm{H}_{3} \mathrm{PO}_{4}\right)$ and $(\mathrm{B})$ acetonitrile. Elution at gradient mode: $0-1 \mathrm{~min}$ : $65 \% \mathrm{~A}$ and $35 \% \mathrm{~B}$; from 1 to 5 min: gradient to $50 \% \mathrm{~A}$ and $50 \% \mathrm{~B}$; from 5 to $5.5 \mathrm{~min}$ : gradient to 65\% $\mathrm{A}$ and $35 \% \mathrm{~B}$; $5.5-7 \mathrm{~min}$ : 65\% $\mathrm{A}$ and $35 \% \mathrm{~B}$. Flow: $1.2 \mathrm{~mL} \mathrm{~min}^{-1}$. A typical chromatogram of this separation can be seen in Fig. 3 . No shift in baseline is apparent, at least for these modest gradient conditions, indicating the absence of significant artefacts such as those caused by refractive index effects. Quantitative data were also obtained to evaluate the detector performance, are summarized in Table 2, and comparable to the results obtained for the isocratic elution.

\section{Conclusion}

It has been successfully demonstrated that a simple UV-detector without monochromator is possible with a $235 \mathrm{~nm}$ LED. Photodiodes were used for light detection, which are less expensive and more straightforward to use than photomultipliers. While these are the less sensitive the shorter the wavelength is, in combination with the deep-UV LED they were found to still perform well 
Table 1

Analytical figure-of-merits obtained for the HPLC LED-detector in isocratic elution mode.

\begin{tabular}{|c|c|c|c|}
\hline & Correlation coefficient for peak area $(\mathrm{r})^{\mathrm{a}}$ & Reproducibility for peak area $(\%)^{b}$ & $\operatorname{LOD}\left(\mathrm{mg} \mathrm{L}^{-1}\right)$ \\
\hline Pioglitazone & 0.9993 & 0.83 & 1.7 \\
\hline Glimepiride & 0.9993 & 0.51 & 0.5 \\
\hline
\end{tabular}

${ }^{c}$ Concentrations corresponding to peaks whose heights are three times the baseline noise.

a For six concentrations (5-100 $\left.\mathrm{mg} \mathrm{L}^{-1}\right)$.

b $\mathrm{RSD}, \mathrm{n}=5$, concentration of $50 \mathrm{mg} \mathrm{L}^{-1}$.

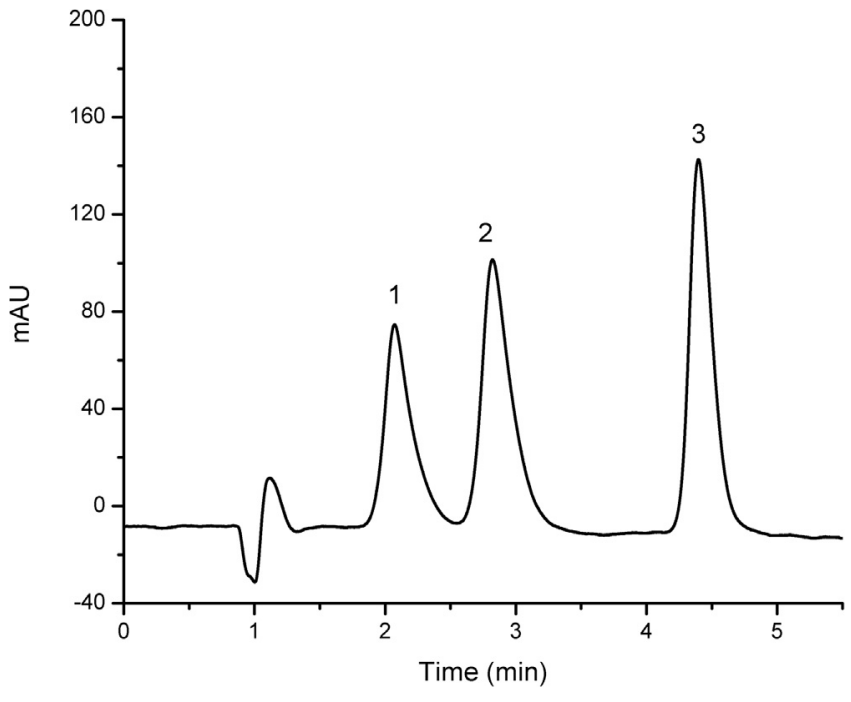

Fig. 3. Separation of benzodiazepines with detection at $235 \mathrm{~nm}$. Standard solution of $50 \mathrm{mg} \mathrm{L}^{-1}$ for each analyte: (1) Flurazepam; (2) Oxazepam; (3) Clobazam. Column: LiChrospher $^{\varpi} 60$-RP-select B, $5 \mu \mathrm{m}, 125 \times 4.6 \mathrm{~mm}$. Mobile phase: $50 \mathrm{mM} \mathrm{KH}_{2} \mathrm{PO}_{4}$ (pH 3.1 with $\left.\mathrm{H}_{3} \mathrm{PO}_{4}\right) / \mathrm{ACN}, \mathrm{t}=0 \mathrm{~min}, 65: 35(\mathrm{v} / \mathrm{v}), \mathrm{t}=1 \mathrm{~min}, 65: 35 ; \mathrm{t}=5 \mathrm{~min}, 50: 50$; $\mathrm{t}=5.5 \mathrm{~min}, 65: 35 ; \mathrm{t}=7 \mathrm{~min}, 65: 35$. Flow rate: $1.2 \mathrm{~mL} \mathrm{~min}^{-1}$. Injected volume: $8 \mu \mathrm{L}$.

\section{Table 2}

Analytical figure-of-merits obtained for the HPLC LED-detector in gradient elution mode.

\begin{tabular}{llll}
\hline & $\begin{array}{l}\text { Correlation } \\
\text { coefficient for } \\
\text { peak area }(\mathrm{r})^{\mathrm{a}}\end{array}$ & $\begin{array}{l}\text { Reproducibility } \\
\text { for peak area }(\%)^{\mathrm{b}}\end{array}$ & LOD $\left(\mathrm{mg} \mathrm{L}^{-1}\right)^{\mathrm{c}}$ \\
\hline Flurazepam & 0.9991 & 0.67 & 1.3 \\
Oxazepam & 0.9988 & 0.86 & 1.3 \\
Clobazam & 0.9998 & 0.52 & 0.8 \\
\hline
\end{tabular}

${ }^{\text {a }}$ For six concentrations $\left(2.5-75 \mathrm{mg} \mathrm{L}^{-1}\right)$.

b $\mathrm{RSD}, \mathrm{n}=5$, concentration of $50 \mathrm{mg} \mathrm{L}^{-1}$.

${ }^{c}$ Concentrations corresponding to peaks whose heights are three times the baseline noise.

for its emission band at $235 \mathrm{~nm}$. The use of this short wavelength enables the detection of compounds which only show absorption bands below $255 \mathrm{~nm}$, which hitherto was the lower wavelength limit accessible with LEDs.

\section{Acknowledgments}

The authors thank Crystal IS for making available the engineering sample of the $235 \mathrm{~nm}$ LED. JFSP and AAC are grateful for the financial support provided by the São Paulo State Research Foundation (FAPESP, No. 2016/16830-0).

\section{References}

[1] P.K. Dasgupta, H.S. Bellamy, H. Liu, J.L. Lopez, E.L. Loree, K. Morris, K. Petersen, K.A. Mir, Light emitting diode based flow-through optical absorption detectors, Talanta 40 (1993) 53-74.
[2] P.K. Dasgupta, I.Y. Eom, K.J. Morris, J. Li, Light emitting diode-based detectors: absorbance, fluorescence and spectroelectrochemical measurements in a planar flow-through cell, Anal. Chim. Acta 500 (2003) 337-364.

[3] D.A. Bui, P.C. Hauser, Analytical devices based on light-emitting diodes - a review of the state-of-the-art, Anal. Chim. Acta 853 (2015) 46-58.

[4] M. Macka, T. Piasecki, P.K. Dasgupta, Light-Emitting diodes for analytical chemistry, Annu. Rev. Anal. Chem. 7 (2014) 183-207.

[5] D.A. Bui, P.C. Hauser, Absorbance measurements with light-emitting diodes as sources: silicon photodiodes or light-emitting diodes as detectors? Talanta 116 (2013) 1073-1078.

[6] D.A. Bui, P.C. Hauser, Absorbance detector for capillary electrophoresis based on light-emitting diodes and photodiodes for the deep-ultraviolet range, J. Chromatogr. A 1421 (2015) 203-208.

[7] T.R. Dias, M.A.S. Brasil, M.A. Feres, B.F. Reis, A flow cell with a new design to improve the utilization of the radiation emitted by LED and employed as a radiation source for photometric detection, Sens. Actuators B Chem. 198 (2014) 448-454

[8] A.A. Cardoso, H. Liu, P.K. Dasgupta, Fluorometric fiber optic drop sensor for atmospheric hydrogen sulfide, Talanta 44 (1997) 1099-1106.

[9] P.C. Hauser, S.S.S. Tan, All-solid-state instrument for fluorescence-based fiberoptic chemical sensors, Analyst 118 (1993) 991-995.

[10] A.E. Bruno, F. Maystre, B. Krattiger, P. Nussbaum, E. Gassmann, The pigtailing approach to optical detection in capillary electrophoresis, Trends Anal. Chem. 13 (1994) 190-198.

[11] P.A.G. Butler, B. Mills, P.C. Hauser, Capillary electrophoresis detector using a light emitting diode and optical fibres, Analyst 122 (1997) 949-953.

[12] J. Ma, S.I. Ohira, S.K. Mishra, M. Puanngam, P.K. Dasgupta, S.B. Mahon, M. Brenner, W. Blackledge, G.R. Boss, Rapid point of care analyzer for the measurement of cyanide in blood, Anal. Chem. 83 (2011) 4319-4324.

[13] X. Yang, J. Du, Y. Zhou, Rapid and point of care measurement of sulfide in human serum with a light emitting diode-based photometer by marriage of gas separation with paper enrichment, Sens. Actuators B Chem. 232 (2016) 738-743.

[14] M. O'Toole, D. Diamond, Absorbance based light emitting diode optical sensors and sensing devices, Sensors 8 (2008) 2453-2479.

[15] G.J. Schmidt, R.P.W. Scott, Simple and sensitive ion chromatograph for trace metal determination, Analyst 109 (1984) 997-1002.

[16] L. Barron, P.N. Nesterenko, D. Diamond, M.O. Toole, K.T. Lau, B. Paull, Low pressure ion chromatography with a low cost paired emitter - detector diode based detector for the determination of alkaline earth metals in water samples, Anal. Chim. Acta 577 (2006) 32-37.

[17] A. Berthod, M. Glick, J.D. Winefordner, Sensitive, indirect photometric detector for high-performance liquid chromatography using a light-emitting diode, J. Chromatogr. 502 (1990) 305-315.

[18] D.A. Bui, B. Bomastyk, P.C. Hauser, Absorbance detector based on a deep UV light emitting diode for narrow-column HPLC, J. Sep. Sci. 36 (2013) 3152-3157.

[19] S. Schmid, M. Macka, P.C. Hauser, UV-absorbance detector for HPLC based on a light-emitting diode, Analyst 133 (2008) 465-469.

[20] B. Bomastyk, I. Petrovic, P.C. Hauser, Absorbance detector for high-performance liquid chromatography based on light-emitting diodes for the deep-ultraviolet range, J. Chromatogr. A 1218 (2011) 3750-3756.

[21] S. Sharma, H.D. Tolley, P.B. Farnsworth, M.L. Lee, LED-based UV absorption detector with low detection limits for capillary liquid chromatography LED-based UV absorption detector with low detection limits for capillary liquid chromatography, Anal. Chem. 87 (2) (2015) 1381-1386.

[22] X. Zhao, X. Xie, S. Sharma, L.T. Tolley, A. Plistil, H.E. Barnett, M. Brisbin, C. Swensen, J.C. Price, P.B. Farnsworth, H.D. Tolley, S.D. Stearns, M.L. Lee, Compact ultra-high pressure nano-flow capillary liquid chromatograph, Anal. Chem. 89 (1) (2017) 807-812.

[23] K.G. Kraiczek, R. Bonjour, Y. Salvade, R. Zengerle, Highly flexible UV-vis radiation sources and novel detection schemes for spectrophotometric HPLC detection, Anal. Chem. 86 (2) (2014) 1146-1152.

[24] Y. Li, P.N. Nesterenko, B. Paull, R. Stanley, M. Macka, Performance of a new $235 \mathrm{~nm}$ UV-LED based on-capillary photometric detector, Anal. Chem. 88 (24) (2016) 12116-12121.

[25] L. Mercolini, R. Mandrioli, M. Amore, M.A. Raggi, Separation and HPLC analysis of 15 benzodiazepines in human plasma, J. Sep. Sci. 31 (2008) 2619-2626.

[26] V. Pandit, R.S. Pai, K. Devi, G. Singh, S. Narayana, S. Suresh, Development and validation of the liquid chromatographic method for simultaneous estimation of metformin pioglitazone, and glimepiride in pharmaceutical dosage forms, Pharm. Methods 3 (2012) 9-13. 